\title{
Comparación de los desenlaces clínicos con cuatro técnicas de apendicetomía laparoscópica tras la implementación de políticas de reúso de dispositivos médicos
}

\author{
Óscar A. Vergara ${ }^{1}$, Luis Carlos Domínguez ${ }^{1}$, Diego Orlando Sierra ${ }^{1}$, \\ Óscar A. Gamboa² y Franco J. Segnini ${ }^{1}$.
}

'Departamento de Cirugía, Campus Biomédico, Colombia. ${ }^{2}$ Departamento de Epidemiología. Universidad de la Sabana, Colombia.

Recibido 2019-07-09 y aceptado 2020-03-03

Correspondencia a: Dr. Luis Carlos Domínguez carlosdot@unisabana.edu.co
Comparison of clinical outcomes with four laparoscopic appendectomy techniques after implementation of medical devices reuse policy

Aim: The present study sought to compare the clinical outcomes between four operative techniques for laparoscopic appendectomy in a Colombian institution, following the adoption of reuse and reprocessing policies for medical devices in the Region of Americas (2014). Materials and Methods: One-way analysis of variance (ANOVA) and Kruskal Wallis tests were used to compare the rates of Surgical Site Infection (SSI), unplanned reoperation, readmission and mortality (confined to 30 days after surgery) of these operative techniques: Technique 1: Ligature of appendiceal stump with endo stitch plus mesenteric dissection with conventional bipolar electrocoagulation; Technique 2: Clipping of stump with polymer endoclip plus mesenteric dissection with conventional bipolar electrocoagulation; Technique 3: Clipping of stump with polymer endoclip plus mesenteric dissection with monopolar electrocoagulation; Technique 4: Clipping of stump plus mesenteric dissection with polymer endoclip. Results: A total of 551 patients were included. No differences were found in the demographic characteristics of the patients between techniques during ANOVAs. The global outcomes were SSI (5.44\%), unplanned reoperation (3.08\%), readmission (15.97\%) and mortality $(0.18 \%)$. Significant differences were found $(\mathrm{p}<0.05)$ in these outcomes explained by higher rates of SSI and unplanned reoperation with Technique 1. Conclusions: The overall outcomes of laparoscopic appendectomy, after the adoption of policies for the reuse and reprocessing of medical devices, are similar to those accepted globally when using operative techniques 2-3-4 in equivalent populations of patients with acute appendicitis. In this context, our results invite to use with caution technique-1.

Key words: laparoscopic appendectomy, reuse; reprocessing; medical devices; clinical outcomes; Colombia.

\section{Resumen}

Objetivo: En este estudio se comparan los desenlaces clínicos de cuatro técnicas de apendicectomía laparoscópica utilizadas en una institución colombiana para el manejo de la apendicitis aguda tras la adopción de políticas de reúso y reprocesamiento de dispositivos médicos para la Región de las Américas (2014). Materiales y Método: Mediante el análisis de varianza de una vía (ANOVA) y la prueba de Kruskal Wallis, se compararon las tasas de infección de sitio operatorio (ISO), reoperación no planeada, readmisión y mortalidad (en los primeros 30 días del postoperatorio) de las siguientes técnicas: Técnica 1: Ligadura del muñón apendicular con endonudo y disección del mesenterio apendicular con electrocoagulación bipolar convencional; Técnica 2: Clipaje del muñón con endoclip de polímero y disección del mesenterio con electrocoagulación bipolar convencional; Técnica 3: Clipaje del muñón con endoclip de polímero y disección del mesenterio con electrocoagulación monopolar; Técnica 4: Clipaje del muñón y disección roma y ligadura del mesenterio con endoclip de polímero.

Resultados: Se incluyeron 551 pacientes. No se encontraron diferencias en los ANOVAs en cuanto a 
las características demográficas de los pacientes por cada técnica. Los desenlaces globales fueron: ISO $(5,44 \%)$, reoperación no planeada $(3,08 \%)$, readmisión $(15,97 \%)$ y mortalidad $(0,18 \%)$. Se encontraron diferencias significativas $(\mathrm{p}<0,05)$ en estos desenlaces explicados por mayores tasas de ISO y reoperación con la Técnica-1. Conclusiones: Los desenlaces globales de la apendicectomía laparoscópica tras la adopción de políticas de reúso de dispositivos médicos se encuentran dentro de los aceptados globalmente cuando se utilizan las Técnicas 2-3-4 en poblaciones equivalentes de pacientes con apendicitis aguda. En este contexto, nuestros resultados invitan a utilizar con precaución la Técnica-1.

Palabras clave: apendicectomía laparoscópica; reúso; reprocesamiento; dispositivos médicos; desenlaces clínicos; Colombia.

\section{Introducción}

La apendicectomía laparoscópica fue introducida hace más de treinta años, y hoy en día es aceptada como la primera opción para el manejo quirúrgico de la apendicitis aguda ${ }^{1,3}$. Colombia no ha sido ajena a esta realidad ${ }^{4-8}$. Los beneficios de esta técnica con respecto a la abierta han sido evidentes, pero también controversiales. Por ejemplo, la menor tasa de infección del sitio operatorio (ISO), complicaciones globales y estancia hospitalaria, y las percepciones sobre la calidad de vida por parte de los pacientes, son algunas de sus ventajas ${ }^{9-11}$. No obstante, el costo y disponibilidad han sido señalados como sus principales limitaciones, ${ }^{9,10,12,13}$.

Los cirujanos han enfrentado estas limitaciones realizando múltiples adaptaciones a la técnica operatoria de acuerdo con el contexto local y disponibilidad de recursos y/o reutilizando la tecnología disponible ${ }^{14,15}$. En contextos como el colombiano, en el que participan intermediarios y terceros pagadores, estas adaptaciones y reutilización de dispositivos permitieron la difusión de la técnica disminuyendo los costos asociados, y evitando los problemas de pago por parte de los aseguradores a las instituciones, a partir de su introducción alrededor del año 2000. Este fenómeno fue observado también en el caso de la colecistectomía laparoscópica y la herniorrafia inguinal ${ }^{16,17}$. La optimización en el uso de recursos se concentró, en particular, en el reúso de los dispositivos de acceso a la cavidad abdominal (trocares), de los dispositivos de energía para el manejo del mesenterio (bisturí ultrasónico y bipolar avanzado) y de los dispositivos para el manejo del muñón apendicular (clips de polímero). Con estos recursos, los resultados clínicos iniciales de la apendicectomía laparoscópica fueron superiores los de la abierta en ${ }^{4-7}$.

No obstante, el sistema de salud colombiano sufrió cambios acelerados a partir de 2014 en materia de estándares internacionales de calidad. En particular, en 2014, se instauró el Mapeo de la Regulación de Reúso y Reprocesamiento de dispositivos médicos en la Región de las Américas, iniciativa de la Organización Panamericana de la Salud (OPS) $)^{18}$ y en 2017, el Instituto Nacional de Vigilancia de Medicamentos y Alimentos de Colombia (INVIMA) socializó las Buenas Prácticas de Manufactura de Dispositivos Médicos, así como la Guía de Reprocesamiento de Dispositivos Médicos, un proyecto necesario para el cumplimiento de los Estándares de Calidad para Habilitación de los Servicios de Salud, de acuerdo a los estándares internacionales (U.S. Department of Health and Human Services Food and Drug Administration $)^{19}$. Como consecuencia, la disminución en la reutilización de dispositivos médicos influyá en la oportunidad y acceso a diferentes procedimientos, incluida la apendicetomía laparoscópica, en el momento en el que incrementaron las tarifas para los pagadores, en especial por los costos derivados de los dispositivos de energía ultrasónica y bipolar avanzada. Estos cambios se dieron de forma paralela a los cambios acelerados en las políticas de regulación de precios, tarifas y competitividad en el sector salud ${ }^{15,20}$. En muchos casos, los aseguradores restringieron la autorización del procedimiento por los sobrecostos y en algunas instituciones se volvieron a realizar apendicectomías abiertas. En otros casos, los sobrecostos fueron asumidos para las instituciones de salud que continuaron realizando apendicectomías laparoscópicas "convencionales" por su superioridad clínica (en especial con dispositivos de energía no reutilizados), cuando estos no fueron asumidos por los aseguradores. Finalmente, en otras instituciones los cirujanos realizaron modificaciones a la técnica quirúrgica laparoscópica, en especial para el manejo del mesenterio y muñón apendicular. Estas modificaciones pretendían estimular la costo-eficiencia, eficacia y utilidad de 
la intervención, y se sustentaron en la literatura disponible, la cual demuestra resultados clínicos equivalentes (complicaciones, reintervención y estancia hospitalaria) cuando se utilizan dispositivos de energía ultrasónica y bipolar avanzada versus la electrocoagulación monopolar o bilopar convencional, ligadura o clips de titanio en el mesenterio apendicular o cuando el muñón apendicular se cierra con diferentes recursos (nudos, suturas, clips $)^{21-24}$.

La información sobre los desenlaces clínicos derivados de este último grupo de adaptaciones es limitada en contextos similares al colombiano. Aún se requieren comparaciones sobre los resultados clínicos tras la introducción de políticas de reúso de dispositivos médicos y el cambio drástico en la técnica quirúrgica, técnica de la apendicectomía laparoscópica. Esta información puede ser útil no solo para los cirujanos e instituciones, sino para los terceros pagadores (aseguradores) y puede permitir la adopción de guías y estándares que estimulen resultados en salud costo-conscientes de alta calidad ${ }^{25}$ en contextos como el latinoamericano, el cual experimenta acelerados cambios en materia de regulaciones y transiciones en sus sistemas de salud.

\section{Materiales y Método}

Este estudio fue aprobado por el Comité de ética de la Clínica Universidad de la Sabana (Colombia). Es un estudio retrospectivo en el que evaluamos una serie de pacientes mayores de 13 años, sometidos a apendicectomía laparoscópica por apendicitis aguda entre mayo de 2016 y junio de 2018. Excluimos los pacientes sometidos a apendicectomía abierta, aquellos referidos de otras instituciones en el periodo posoperatorio y los que presentaban datos incompletos. Diseñamos una base de datos en la que incluimos variables sociodemográficas (edad, género), clínicas (tiempo de evolución de los síntomas, antecedentes médico quirúrgicos, puntaje en la Escala de Alvarado, puntaje de la clasificación de la American Society of Anaestesiology y tiempo entre el diagnóstico de apendicitis y el inicio de la cirugía) y operatorias (tiempo quirúrgico, fase de evolución de la apendicitis y recursos tecnológicos utilizados para el manejo del mesenterio y muñón apendicular). Obtuvimos la información a partir de los registros sistematizados de la historia clínica electrónica.

De forma general, los procedimientos fueron practicados por cirujanos con experiencia en ci- rugía de mínima invasión abdominal, mediante técnica de acceso abierto umbilical a cavidad, neumoperitoneo de $14 \mathrm{mmHg}$ e introducción de tres trocares. En todos los casos se administró antibiótico profiláctico, de acuerdo con los estándares del Center for Disease Control and Prevention $(\mathrm{CDC})^{26}$.

De acuerdo con la información, inicialmente identificamos y categorizamos las diferentes técnicas operatorias, resultantes de la combinación de recursos para el manejo del mesenterio y muñón apendicular. Las definiciones adoptadas para los dispositivos tecnológicos de hemostasia fueron adoptadas a partir de las convenciones señaladas en la literatura científica ${ }^{27}$. Posteriormente, comparamos las variables sociodemográficas y clínicas entre los grupos de manejo quirúrgico previamente identificados con el fin de identificar la homogeneidad de las poblaciones. Finalmente, evaluamos los desenlaces clínicos relacionados con cada una de las técnicas: a) número y porcentaje de pacientes con infección de sitio operatorio (ISO), de acuerdo a los criterios de Center for Disease Control and Prevention $(\mathrm{CDC})^{26}$; b) número y porcentaje de pacientes con readmisión al urgencias por cualquier causa durante los primeros 30 días del postoperatorio; c) número y porcentaje de pacientes sometidos a reoperación no planeada durante los primeros 30 días de postoperatorio; 4) estancia hospitalaria (horas), definida como el periodo comprendido entre el ingreso al servicio de urgencias y el egreso institucional y 5) tasa de mortalidad (confinada a los primeros 30 días del posoperatorio).

Describimos las variables cuantitativas mediante promedios y desviación estándar cuando los datos siguieron una distribución simétrica. En los casos con distribuciones asimétricas utilizamos la mediana y el rango intercuartílico (RIQ) como medidas de tendencia central y dispersión, respectivamente. Las variables cualitativas fueron descritas usando frecuencias absolutas y relativas. Determinamos el balance de los grupos de acuerdo con las características de base usando el análisis de varianza de una vía (ANOVA) en las variables cuantitativas si se cumplía el supuesto de normalidad, o su equivalente no paramétrico, la prueba de Kruskal Wallis, si no se cumplía este supuesto. Para las variables categóricas utilizamos la prueba de independencia Chi cuadrado. En la valoración de los desenlaces utilizamos las pruebas anteriormente descritas de acuerdo con la naturaleza de las variables. Realizamos los análisis a dos colas para un nivel de significancia del 0,05. Utilizamos el programa Stata 14. 


\section{Resultados}

De un total de 1.247 pacientes operados por apendicitis aguda entre mayo de 2016 y julio de 2018, 551 cumplieron con los criterios del estudio y fueron incluidos en el análisis. Los 696 pacientes restantes fueron sometidos a apendicetomía abierta.

\section{Técnica operatoria}

Identificamos tres recursos para el manejo del mesenterio apendicular: 1) disección del mesenterio apendicular con electrocoagulación monopolar; 2) disección del mesenterio apendicular con electrocoagulación bipolar convencional; 3) disección roma y ligadura del mesenterio con endoclip de polímero. Identificamos dos recursos para el manejo del muñón apendicular: 1) ligadura del muñón apendicular con endonudo y 2) clipaje del muñón apendicular con endoclip de polímero. Finalmente, identificamos cuatro posibles resultantes de la combinación de estos recursos:

- Técnica 1: Ligadura del muñón apendicular con endonudo y disección del mesenterio apendicular con electrocoagulación bipolar convencional: 61 pacientes $(11,07 \%)$

- Técnica 2: Clipaje del muñón apendicular con endoclip de polímero y disección del mesenterio apendicular con electrocoagulación bipolar convencional: 64 pacientes $(11,61 \%)$.

- Técnica 3: Clipaje del muñón apendicular con endoclip de polímero y disección del mesenterio apendicular con electrocoagulación monopolar: 104 pacientes $(18,87 \%)$.

- Técnica 4: Clipaje del muñón apendicular con endoclip de polímero y disección roma y ligadura del mesenterio con endoclip de polímero: 322 pacientes $(58,43 \%)$.

Las características demográficas y clínicas de los pacientes por cada técnica se presentan en la Tabla 1. En cuanto a las variables operatorias, encontramos diferencias estadísticamente significativas en el tiempo operatorio entre las cuatro combinaciones, desde 43,90 \pm 19,80 min (Técnica 4) hasta 53,83 \pm 21,57 min (Técnica 1).

\section{Desenlaces clínicos}

El porcentaje global de ISO fue 5,44\% (30 pacientes). Identificamos un total de 88 readmisiones al servicio de urgencias $(15,97 \%)$ durante los primeros 30 días del periodo posoperatorio. El 3,08\% (17 pacientes) fueron sometidos a reoperación no planeada. Estos tres desenlaces (ISO, reingreso y reoperación no planeada) fueron superiores con la
Técnica 1 (Tabla 2). No identificamos diferencias en la tasa de estancia hospitalaria entre las cuatro técnicas. La tasa de mortalidad fue $0,18 \%$ (1 paciente). Los desenlaces clínicos por cada técnica operatoria se presentan en la Tabla 2.

\section{Discusión}

En el presente estudio evaluamos los desenlaces clínicos de diferentes técnicas operatorias para el manejo de la apendicitis aguda en el contexto de la Regulación de reúso y reprocesamiento de dispositivos médicos en la Región de las Américas, iniciativa de la Organización Panamericana de la Salud (OPS), adoptada en Colombia a partir del año 2014. En la serie de casos identificamos cuatro técnicas operatorias. La Técnica 4 fue utilizada con mayor frecuencia (clipaje del muñón apendicular con endoclip de polímero asociado a la disección roma y ligadura del mesenterio con endoclip de polímero). La Técnica 1 fue utilizada con menor frecuencia (ligadura del muñón apendicular con endonudo asociado a la disección del mesenterio apendicular con electrocoagulación bipolar convencional). Las características sociodemográficas y clínicas de la población no demostraron diferencias entre las cuatro técnicas. Un segundo hallazgo indica que las variables operatorias y los desenlaces globales fueron similares a los reportados en la literatura ${ }^{1,4,5,28,29}$ (LA; no obstante, el tiempo quirúrgico, el porcentaje de ISO, la readmisión al servicio de urgencias y la reoperación no planeada fueron superiores con la Técnica 1 .

Estos hallazgos tienen varias explicaciones. En primer lugar, el uso frecuente de la Técnica 4 puede explicarse por la amplia disponibilidad y bajo costo de recursos como el endoclip de polímero en el contexto de regulación de reúso de dispositivos médicos. Antes de 2014, la apendicectomía laparoscópica tuvo un desarrollo acelerado en Colombia a través del uso de dispositivos de energía ultrasónica y bipolar avanzada. Creemos que, tras la introducción de la normatividad y la regulación en el uso de dispositivos de alto costo, los cirujanos se vieron expuestos a utilizar técnicas alternas más económicas, mientras garantizaban resultados clínicos equivalentes. Este fenómeno ha sido descrito previamente ${ }^{15}$. El presente estudio caracteriza este tipo de técnicas, en especial las que requieren dispositivos de clipaje mecánico (Técnica 4), dispositivos de energía convencional (bipolar convencional o monopolar) (Técnicas 2-3) o ligaduras intracorpóreas (Técnica 1). Paralelamente, creemos que la 
Tabla 1. Características demográficas y análisis de varianza de una vía (ANOVA) en las variables cuantitativas de la población por cada una de las técnicas

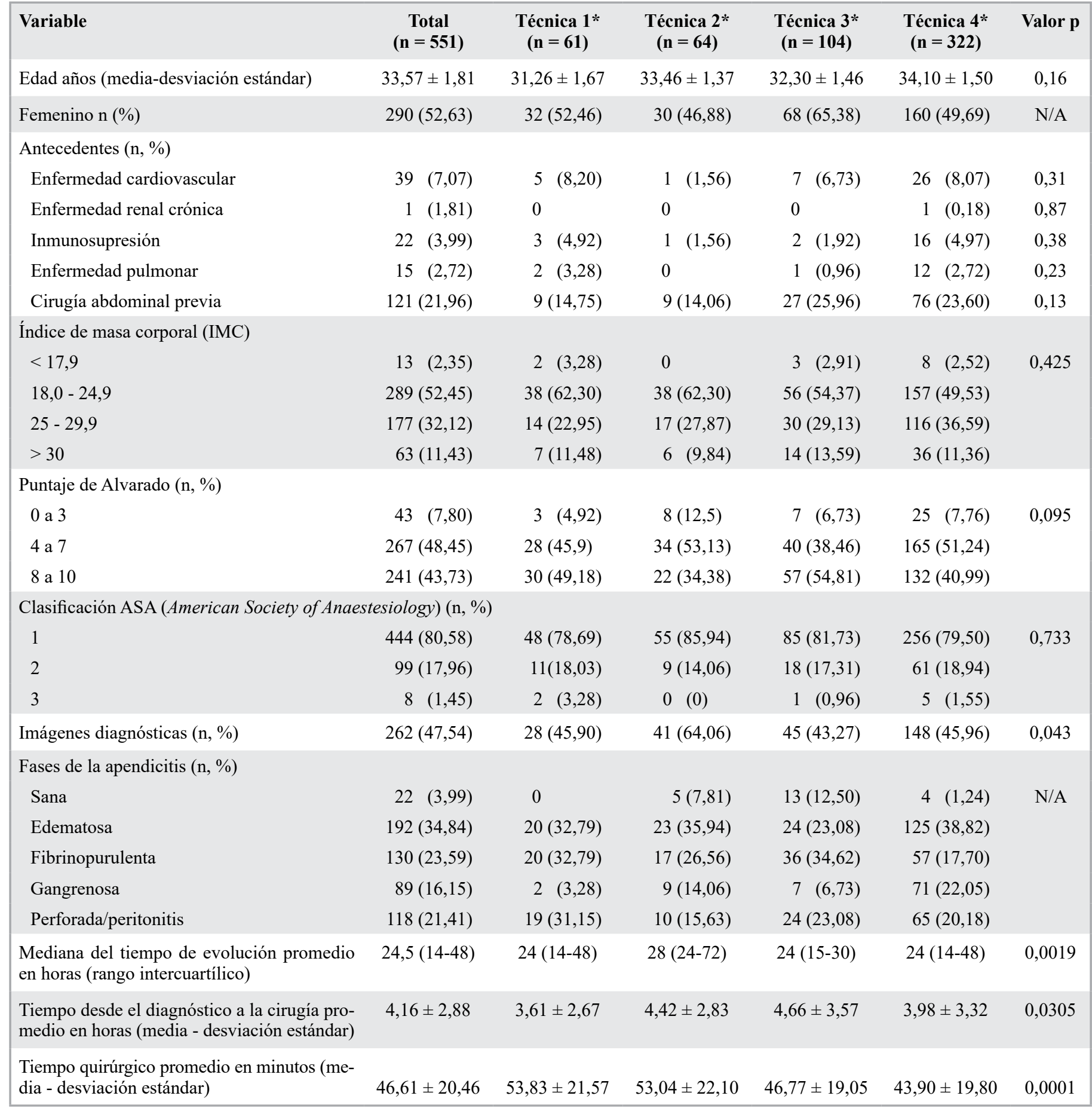

*Técnica 1: Ligadura del muñón apendicular con endonudo y disección del mesenterio apendicular con electrocoagulación bipolar convencional. Técnica 2: Clipaje del muñón apendicular con endoclip de polímero y disección del mesenterio apendicular con electrocoagulación bipolar convencional. Técnica 3: Clipaje del muñón apendicular con endoclip de polímero y disección del mesenterio apendicular con electrocoagulación monopolar. Técnica 4: Clipaje del muñón apendicular con endoclip de polímero y disección roma y ligadura del mesenterio con endoclip de polímero. N/A: No aplica (no se cumple el supuesto del número de observaciones por celda para la prueba Chi cuadrado). 
ARTíCULO ORIGINAL

Tabla 2. Análisis de varianza de una vía (ANOVA) en los desenlaces clínicos (confinados a los primeros 30 días del período posoperatorio) por cada una de las técnicas

\begin{tabular}{|c|c|c|c|c|c|c|}
\hline Variable & $\begin{array}{c}\text { Total } \\
(n=551)\end{array}$ & $\begin{array}{l}\text { Técnica } 1^{*} \\
(\mathrm{n}=61)\end{array}$ & $\begin{array}{l}\text { Técnica } 2 * \\
(n=64)\end{array}$ & $\begin{array}{l}\text { Técnica } 3 * \\
(n=104)\end{array}$ & $\begin{array}{l}\text { Técnica } 4^{*} \\
(\mathrm{n}=322)\end{array}$ & Valor $\mathbf{p}$ \\
\hline $\begin{array}{l}\text { Media estancia hospitalaria en horas } \\
\text { (rango intercuartílico) }\end{array}$ & & $\begin{array}{c}21 \\
(13-60)\end{array}$ & $\begin{array}{c}32 \\
(16,5-70)\end{array}$ & $\begin{array}{c}26 \\
(18-67,5)\end{array}$ & $\begin{array}{c}31,5 \\
(18-75)\end{array}$ & 0,0887 \\
\hline Reoperación no planeada (n, \%) & $17(3,08)$ & $7(11,48)$ & 0 & 0 & $10(3,11)$ & $<0,001$ \\
\hline $\begin{array}{l}\text { Reingreso a urgencias (primeros } 30 \text { días } \\
\text { del posoperatorio) (n, \%) }\end{array}$ & $88(15,97)$ & $16(26,23)$ & $8(12,50)$ & $12(11,54)$ & $52(16,15)$ & 0,076 \\
\hline Infección del sitio operatorio (ISO) (n, \%) & $30(5,44)$ & $10(16,39)$ & $1(1,56)$ & $1(0,96)$ & $18(5,59)$ & \multirow{4}{*}{$<0,001$} \\
\hline Superficial & $7(1,27)$ & $1(1,64)$ & 0 & 0 & $6(1,86)$ & \\
\hline Profunda & $4(0,72)$ & $1(1,64)$ & 0 & $1(0,96)$ & $2(0,62)$ & \\
\hline Órgano/espacio & $19(3,44)$ & $8(13,11)$ & $1(1,56)$ & 0 & $10(3,11)$ & \\
\hline Mortalidad (n, \%) & $1(0,18)$ & $1(1,6)$ & 0 & 0 & 0 & 0,1 \\
\hline
\end{tabular}

*Técnica 1: Ligadura del muñón apendicular con endonudo y disección del mesenterio apendicular con electrocoagulación bipolar convencional. Técnica 2: Clipaje del muñón apendicular con endoclip de polímero y disección del mesenterio apendicular con electrocoagulación bipolar convencional. Técnica 3: Clipaje del muñón apendicular con endoclip de polímero y disección del mesenterio apendicular con electrocoagulación monopolar. Técnica 4: Clipaje del muñón apendicular con endoclip de polímero y disección roma y ligadura del mesenterio con endoclip de polímero.

mayor utilización de estas técnicas puede explicarse por la influencia de la formación especializada en los programas de residencia. Durante los últimos cinco años, en los hospitales universitarios y centros académicos colombianos, el entrenamiento de nuevas generaciones de cirujanos ha generalizado el uso de diversas técnicas de "bajo costo" (como las previamente descritas), y muchos profesionales las han integrado a su práctica de forma rutinaria con resultados clínicos satisfactorios. La necesidad de estudios en este campo es parte de la justificación de nuestra investigación.

En el segundo hallazgo identificamos resultados clínicos globales similares a los reportados en la literatura internacional. Por ejemplo, el consenso de la Sociedad Europea de Cirugía Endoscópica (EAES) de 2015 demostró un porcentaje de ISO superficial y profunda que oscila entre $1,2 \%$ y $12 \%$, respectivamente, y de ISO órgano/espacio que varía entre $1,6 \%$ y $8 \%{ }^{2}$. Igualmente, en estudios norteamericanos que analizaron series nacionales de más de cien mil pacientes sometidos a apendicectomía ( $92 \%$ correspondientes a apendicectomía laparoscópica), se identificó un porcentaje global de ISO cercano al 3,4\% (ISO órgano/espacio 2,3\%) ${ }^{1}$, el cual es cercano a $8,8 \%$ en países en vías de desarro$1 \mathrm{o}^{29}$. Estos estudios permiten identificar una tasa de mortalidad inferior a $0,1 \%$ y de readmisión y reoperación no planeada, inferiores al 3\% ${ }^{1}$. La literatura local también permite establecer comparaciones. En Colombia se reportan series con un porcentaje global de ISO que varia entre el $1,1 \%$ y el $4,62 \% \%^{5-7}$ y tasas de reoperación no planeada y readmisión de $0,77 \%$ y $10 \%$, respectivamente 5 . Sin embargo, estos estudios no reportan resultados discriminados de acuerdo con diferentes tipos de técnicas y recursos. A su vez, un estudio nacional que evaluó a 65.625 pacientes sometidos a apendicectomía (7\% por técnica laparoscópica), reportó un porcentaje de mortalidad inferior al $1 \%$, así como tasas de estancia hospitalaria global de 3,83 días en promedio ${ }^{4}$. Con base en esta información, creemos que nuestros resultados son similares a los de estudios realizados en poblaciones con condiciones iguales de acceso al servicio de salud.

No obstante, identificamos un porcentaje superior de desenlaces negativos con la Técnica 1 , en particular de ISO global, el cual puede explicar el mayor número de readmisiones y a su vez de reoperaciones no planeadas. Estos resultados invitan a revisar la técnica quirúrgica utilizada, si bien las características sociodemográficas y otras variables, operatorias y no operatorias, son similares entre los cuatro grupos. Evidencia preliminar soporta desenlaces clínicos favorables con esta técnica, que son equivalentes a otras técnicas que involucran diferentes recursos tecnológicos. Por ejemplo, se han reportado tasas de ISO órgano/espacio de 1,4\% con el uso de endonudos y nudos extracorpóreos, así como tiempos quirúrgicos similares ${ }^{22,30}$.

Este estudio cuenta con fortalezas y limitaciones. Ofrece un escenario apropiado para realizar un 
análisis comparativo, dado que permite analizar el uso de diferentes recursos tecnológicos utilizados por un grupo de cirujanos en una institución de salud colombiana para el manejo de una patología altamente prevalente. Aunque existen estudios preliminares en poblaciones equivalentes ${ }^{31,32}$, teniendo en cuenta las características propias del sistema sanitario, este es el primer estudio colombiano que compara diferentes recursos tecnológicos en el contexto de las políticas de reúso de dispositivos médicos instauradas en el país. De igual forma, este estudio contribuye al análisis de la calidad de atención y aporta al diseño de estrategias de mejoramiento interno. Reconocemos que este es un análisis retrospectivo que carece de una evaluación de costo/ efectividad de intervenciones. Invitamos a realizar nuevos estudios al respecto.

Este estudio tiene implicaciones prácticas y ofrece oportunidades de investigación futura. Nuestros resultados pueden ser un referente para estudios futuros que evalúen desenlaces clínicos, así como análisis de costos en el tratamiento de la apendicitis aguda en contextos en los que existen regulaciones crecientes en la reutilización de dispositivos médicos. De acuerdo con los resultados del presente estudio, recomendamos el uso seguro de tres técnicas identificadas. Invitamos a utilizar con precaución la Técnica 1, aunque, como hemos mencionado, nuestros resultados obligan a conducir nuevos estudios que permitan explicar con mayor precisión los desenlaces subóptimos y revisar los aspectos técnicos del procedimiento. Finalmente, consideramos que este estudio abre la puerta para nuevas investigaciones, aparte de las ya mencionadas, involucrando variables de impacto económico, así como estudios cualitativos que exploren las percepciones de los cirujanos respecto a las implicaciones que ha traído el cambio de política de reúso de dispositivos médicos en su práctica profesional, en contextos como el colombiano y latinoamericano.

En el presente artículo concluimos que la apendicectomía laparoscópica realizada en una institución colombiana adherida a la normatividad vigente de reúso de dispositivos médicos es segura y comparable con la experiencia de otros contextos. Los resultados tienen implicaciones para la práctica y ofrecen oportunidades de investigación futura.

\section{Responsabilidades éticas}

Protección de personas y animales. Los autores declaran que para esta investigación no se han realizado experimentos en seres humanos ni en animales.

Confidencialidad de los datos. Los autores declaran que en este artículo no aparecen datos de pacientes.

Conflictos de interés: no hay.

\section{Bibliografía}

1. Alore EA, Ward JL, Todd SR, Wilson CT, Gordy SD, Hoffman MK, et al. Population-level outcomes of early versus delayed appendectomy for acute appendicitis using the American College of Surgeons National Surgical Quality Improvement Program. J Surg Res [Internet]. 2018;229:234-42. Available from: https://linkinghub.elsevier.com/ retrieve/pii/S0022480418302506 [citado el 7 de julio de 2019].

2. Gorter RR, Eker HH, Gorter-Stam MAW, Abis GSA, Acharya A, Ankersmit M, et al. Diagnosis and management of acute appendicitis. EAES consensus development conference 2015. Surg Endosc [Internet]. 2016;30(11):4668-90. Available from: http://www.ncbi.nlm.nih. gov/pubmed/27660247 [citado el 7 de marzo de 2019].
3. Ferris M, Quan S, Kaplan BS, Molodecky N, Ball CG, Chernoff GW, et al. The Global Incidence of Appendicitis. Ann Surg [Internet]. 2017;266(2):237-41. Available from: http://www.ncbi.nlm.nih. gov/pubmed/28288060 [citado el 7 de octubre de 2018].

4. Buitrago G, Junca E, Eslava-Schmalbach J, Caycedo R, Pinillos P, Leal LC. Clinical Outcomes and Healthcare Costs Associated with Laparoscopic Appendectomy in a Middle-Income Country with Universal Health Coverage. World J Surg [Internet]. 2018; Available from: http://link.springer.com/10.1007/ s00268-018-4777-5 [citado el 7 de octubre de 2018]

5. Ruiz-Patiño A, Rey S, Molina G, Domínguez LC, Rugeles S. Costeffectiveness of laparoscopic versus open appendectomy in developing nations: a Colombian analysis. J Surg Res [Internet].
2018;224:33-7. Available from: https:// linkinghub.elsevier.com/retrieve/pii/ S0022480417306984 [citado el 7 de octubre de 2018].

6. Mosquera M, Kadamani A, Pacheco M, Villarreal R, Ayala JC, Fajardo LP, et al. Laparoscopic appendectomy versus open appendectomy. Rev Colomb Cirugía 2012;27:121-8.

7. Toro JP, Barrera ÓJ, Morales CH. Superioridad clínica de la apendicectomía laparoscópica sobre la técnica abierta: ¿adopción lenta de un nuevo estándar de tratamiento? Rev Colomb Cir. 2017;32:32-9.

8. Sanabria A, Domínguez LC, Bermúdez C, Serna A. Evaluation of diagnostic scales for appendicitis in patients with lower abdominal pain. Biomedica [Internet]. 2007;27(3):419-28. Available from: http:// www.ncbi.nlm.nih.gov/pubmed/18320107 [citado el 7 de octubre de 2018]. 
9. Haas L, Stargardt T, Schreyoegg J. Costeffectiveness of open versus laparoscopic appendectomy: A multilevel approach with propensity score matching. Eur J Heal Econ. 2012;13:549-60.

10. Beg MA, Faridi S, Qazi AR, Siddiqui F. Laparoscopic appendectomy-comparison with open. Pakistan J Surg. 2017;33:1158.

11. Sanabria A, Vega V, Domínguez LC, Espitia E, Serna A, Osorio C. The evolution of laparoscopy in abdominal surgery: A meta-analysis of the effect on infectious outcomes. Minim Invasive Ther Allied Technol [Internet]. 2014;23(2):7486. Available from: http://www.ncbi.nlm. nih.gov/pubmed/24236695 [citado el 7 de octubre de 2018].

12. Domínguez LC, Sanabria A, Vega V, Osorio C. Early laparoscopy for the evaluation of nonspecific abdominal pain: a critical appraisal of the evidence. Surg Endosc [Internet]. 2011;25(1):108. Available from: http://link.springer. com/10.1007/s00464-010-1145-4 [citado el 7 de octubre de 2018].

13. Sohn M, Agha A, Bremer S, Lehmann KS, Bormann M, Hochrein A. Surgical management of acute appendicitis in adults: A review of current techniques. Int J Surg [Internet]. 2017;48:232-9. Available from: http://www.ncbi.nlm.nih. gov/pubmed/29155250 [citado el 8 de julio de 2019].

14. Dapri G, Casali L, Bruyns J, Himpens J, Cadiere G-B. Single-access laparoscopic surgery using new curved reusable instruments: initial hundred patients. Surg Technol Int [Internet]. 2010;20:21-35. Available from: http://www.ncbi.nlm.nih. gov/pubmed/21082545 [citado el 17 de enero de 2019].

15. Avansino JR, Goldin AB, Risley $\mathrm{R}$, Waldhausen JHT, Sawin RS. Standardization of operative equipment reduces cost. J Pediatr Surg [Internet]. 2013;48:1843-9. Available from: https:// linkinghub.elsevier.com/retrieve/pii/ S0022346812009657 [citado el 17 de enero de 2019].

16. Pinzón FE, Domínguez LC, Rincón FM, Espitia EL. Cost-effective of Laparoscopic Versus Open Groin Hernia Surgery in Colombia: An Institutional Perspective. J Surg Res [Internet]. 2012;172:289. Available from: https:// linkinghub.elsevier.com/retrieve/pii/ S0022480411014211 [citado el 17 de enero de 2019].

17. Fajardo R, Valenzuela JI, Olaya SC, Quintero G, Carrasquilla G, Pinzón CE, et al. [Cost-effectiveness of laparoscopic versus open cholecystectomy]. Biomedica [Internet];31:514-24. Available from: http://www.ncbi.nlm.nih.gov/ pubmed/22674362 [citado el 17 de enero de 2019].

18. Enríquez N, Álvarez Y, María Martínez D, Pérez A, Lemgruber A. Situación de la regulación de los dispositivos médicos en la Región de las Américas Investigación original/Original research [Internet]. Vol. 39, Pan American Journal of Public Health. 2016. Available from: http://iris.paho.org/xmlui/ bitstream/handle/123456789/28529/ v39n5a6 238-244. pdf?sequence $=1 \&$ isAllowed $=y$ [citado el 7 de julio de 2019].

19. Nania P. New guidance for reprocessing medical devices. AORN J [Internet]. 2015;102:P13-4. Available from: http:// www.ncbi.nlm.nih.gov/pubmed/26665909 [citado el 7 de julio de 2019].

20. Fernando R, Leticia A-B, Antonio J. T. How does managed competition affect hospital prices in a social health insurance system? The Colombian case. Health Policy Plan [Internet]. 2018;33:1037-46. Available from: http://www.ncbi.nlm.nih. gov/pubmed/30462221 [citado el $17 \mathrm{de}$ enero de 2019].

21. Shaikh FM, Bajwa R, McDonnell CO Management of appendiceal stump in laparoscopic appendectomy-clips or ligature: a systematic review and metaanalysis. J Laparoendosc Adv Surg Tech A [Internet]. 2015;25:21-7. Available from: http://www.liebertpub.com/doi/10.1089/ lap.2014.0470 [citado el 7 de octubre de 2018].

22. Agalar C, Derici S, Çevlik AD, Aksoy SÖ, Egeli T, Boztaş N, et al. Do the stump knotting technique and specimen retrieval method effect morbidity in laparoscopic appendectomy? Ulus Travma Acil Cerrahi Derg [Internet]. 2019;25:34-8. Available from: http://www.tjtes.org/eng/jvi. aspx?pdir=travma\&plng=eng\&un= UTD90382 [citado el 7 de marzo de 2019].

23. Antoniou SA, Mavridis D, Hajibandeh S,
Hajibandeh S, Antoniou GA, Gorter R, et al. Optimal stump management in laparoscopic appendectomy: A network metaanalysis by the Minimally Invasive Surgery Synthesis of Interventions and Outcomes Network. Surgery [Internet]. 2017;162:994-1005. Available from: https://linkinghub.elsevier.com/retrieve/ pii/S003960601730483X [citado el 7 de octubre de 2018].

24. Amortegui JD, Restrepo H. Knot security in laparoscopic surgery. Surg Endosc [Internet]. 2002;16:1598-602. Available from: http://www.ncbi.nlm.nih.gov/ pubmed/12085155 [citado el 7 de octubre de 2018].

25. Hood VL, Weinberger SE. High value, cost-conscious care: An international imperative. Eur J Intern Med [Internet]. 2012;23:495-8. Available from: http:// www.ncbi.nlm.nih.gov/pubmed/22863424 [citado el 17 de enero de 2019].

26. Mangram AJ, Horan TC, Pearson ML, Silver LC, Jarvis WR. Guideline for Prevention of Surgical Site Infection, 1999. Centers for Disease Control and Prevention (CDC) Hospital Infection Control Practices Advisory Committee. Am J Infect Control [Internet]. 1999;27:97-132; quiz 133-4; discussion 96. Available from: http://www.ncbi.nlm. nih.gov/pubmed/10196487 [citado el 17 de enero de 2019].

27. Balagué C. [Hemostasis and technology. Energy. Development of new technologies]. Cir Esp [Internet]. 2009;85 Suppl 1:15-22. Available from: https:// linkinghub.elsevier.com/retrieve/pii/ S0009739X09716239 [citado el 17 de enero de 2019].

28. Sartelli M, Baiocchi GL, Di Saverio S, Ferrara F, Labricciosa FM, Ansaloni L, et al. Prospective Observational Study on acute Appendicitis Worldwide (POSAW). World J Emerg Surg [Internet]. 2018;13(1):19. Available from: https:// wjes.biomedcentral.com/articles/10.1186/ s13017-018-0179-0 [citado el 7 de octubre de 2018].

29. Foster D, Kethman W, Cai LZ, Weiser TG, Forrester JD. Surgical Site Infections after Appendectomy Performed in Low and Middle Human Development-Index Countries: A Systematic Review. Surg Infect (Larchmt) [Internet]. 2018;19:237- 


\section{ARTí́CULO ORIGINAL}

44. Available from: http://www.ncbi.nlm. nih.gov/pubmed/29058569 [citado el 8 de julio de 2019].

30. Lucchi A, Berti P, Grassia M, Siani LM, Gabbianelli C, Garulli G. Laparoscopic appendectomy: Hem-o-lok versus Endoloop in stump closure. Updates Surg [Internet]. 2017;69:61-5. Available from: http://link.springer.com/10.1007/s13304-
016-0413-9 [citado el 7 de marzo de 2019].

31. Borraez B, Apolinar A, Mateus C, Rodríguez M, Medina M, Romero J, et al. Diagnóstico de la apendicitis y su variación en el tiempo. Rev Chil Cirugía [Internet]. 2019;71. Available from: https://www.revistacirugia.cl/index.php/ revistacirugia/article/view/197 [citado el 7 de julio de 2019].

32. Caballero A, Pinilla MI, Mendoza ICS, Peña JRA. Frecuencia de reingresos hospitalarios y factores asociados en afiliados a una administradora de servicios de salud en Colombia. Cad Saude Publica [Internet]. 2016;32. Available from: http:// www.ncbi.nlm.nih.gov/pubmed/27462855 [citado el 7 de octubre de 2018]. 